\title{
Preface: Groups and Interaction
}

Volume 2 of the book focuses on the second core factor, namely, "groups and interaction," which consists of four chapters. Chapter 1 is about the analysis of user behaviors, Chapter 2 is about the social network sentiment analysis, Chapter 3 is about the individual influence analysis and its techniques, and Chapter 4 is about the group aggregation and influence mechanism.

The following experts and scholars who participated in the data collection, content arrangement, and achievement contribution of this volume are sincerely appreciated: Zhaoyun Ding, Xiaomeng Wang, Bin Wang, Yezheng Liu, Xiaodong Liu, Shenghong Li, Aiping Li, Lei Li, Shiyu Du, Peng Wu, Xiuzhen Chen, Wei Chen, Yang Yang, Lumin Zhang, Peng Shi, and Yuanchun Jiang.

Thanks to Associate Professor Shudong Li for the careful coordination and arrangement for writing this volume, and also to Weihong Han and Shuqiang Yang for reviewing and proofreading. 
